\section{Do all aromatase inhibitors have similar efficacy and safety?}

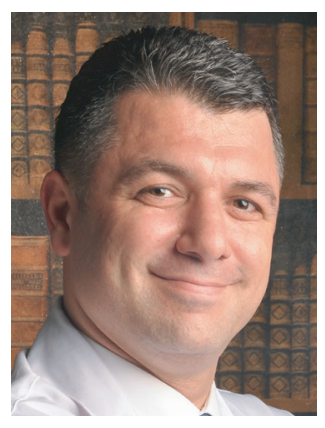

\author{
Mehmet AN Șendur ${ }^{*, 1}$, Burak Bilgin', Mutlu Hızal', Nuriye Yıldırım Özdemir', \\ Muhammed Bülent Akıncı', Didem Șener Dede' \& Bulent Yalçın
}

First draft submitted: 11 May 2017; Accepted for publication: 1 June 2017; Published online: 23 August 2017

Patients with invasive breast cancers that estrogen receptor and/or progesterone receptor are positive should be treated with adjuvant endocrine therapy regardless of stage of the disease, patient age, prior chemotherapy and menopausal status [1]. In the adjuvant hormonal treatment of postmenopausal breast cancer patients, most of the trials have showed the superiority of aromatase inhibitors over tamoxifen. In a meta-analyses of breast cancer outcomes in adjuvant trials of aromatase inhibitors versus tamoxifen, aromatase inhibitors significantly lower recurrence rates compared with tamoxifen treatment either as initial monotherapy or after 2-3 years of tamoxifen [2]. In a metanalyses of 31,920 postmenopausal patients, 5 years of an aromatase inhibitor reduces recurrence rates by about $30 \%$ and 10 -year breast cancer mortality rates by about $15 \%$ compared with 5 years of tamoxifen [3]. Due to the better progression-free survival rate and lower recurrences with aromatase inhibitors compared with tamoxifen in early breast cancer, aromatase inhibitors have been accepted as first-line treatment in the adjuvant treatment of hormone receptor-positive postmenopausal breast cancer [4] .

Letrozole is a more potent inhibitor of aromatase than anastrozole at their conventionally used doses, and leads to more complete inhibition of whole-body aromatase. Geisler $e t$ al. reported that mean percentage aromatase inhibition with anastrozole was $97.1 \%$, whereas more than $99.1 \%$ suppression of aromatase enzyme was observed with letrozole in standard treatment doses [5]. In postmenopausal patients, a randomized Phase II ACOSOG Z1031 trial (NCT00265759) showed that all three aromatase inhibitors; letrozole, anastrozole and exemestane have similar rates of clinical response in the neoadjuvant setting [6]. However, aromatase inhibitors have been accepted as first-line treatment of postmenopausal hormone receptor-positive early breast cancer, it is wondering that which aromatase inhibitor is better than another. Recent published trials investigated the clinical efficacy of aromatase inhbitors.

In a randomized Phase III MA.27 trial (NCT00066573), the efficacy of steroidal aromatase inhibitor exemestane and nonsteroidal aromatase inhibitor anastrozole for 5 years in the adjuvant hormonal treatment of hormone receptor-positive postmenopausal early breast cancer was compared [7]. Total of 7576 patients were enrolled in this first comparison of steroidal and nonsteroidal

'Department of Medical Oncology, Faculty of Medicine, Yıldırım Beyazıt University, Bilkent, Ankara 06800, Turkey

*Author for correspondence: Tel.: +90 312 2912525; Fax: +90 312 2912726; masendur@yahoo.com.tr

\section{KEYWORDS}

- aromatase inhibitors

- breast cancer $\bullet$ endocrine treatment 
classes of aromatase inhibitors trial, and the primary outcome 4-year event-free survival (EFS) rates were similar: 91\% for exemestane and $91.2 \%$ for anastrozole in the initial adjuvant therapy of hormone receptor-positive breast cancer with a median 4.1-year follow-up (HR: $1.02 ; 95 \%$ CI: $0.87-1.18 ; \mathrm{p}=0.85$ ). In a recent published randomized Phase III Femara Versus Anastrozole Clinical Evaluation (FACE) trial (NCT00248170), Smith et al. compared the efficacy and safety of adjuvant letrozole and anastrozole in postmenopausal patients with hormone receptor and node-positive early breast cancer [8]. A total of 4136 patients were randomized and 5-year estimated disease-free survival (DFS) rates were 84.9 and $82.9 \%$ for letrozole and anastrozole arms, respectively (HR: 0.93; 95\% CI: 0.80-1.07; $\mathrm{p}=0.31$ ).

Before we comment that all aromatase inhibitors have similar efficacy in the adjuvant treatment of hormone receptor-positive postmenopausal breast cancer, we should remark some confounding factors that can effect the primary outcome DFS or EFS in both trials. In MA.27 trial, patients stratified according to the positive lymph node status or not [7]. In FACE trial, patients were stratified according to the number of lymph nodes (one to three or four or more) and human EGF receptor 2 (HER2) status. It is well known that both nodal involvement and increased tumor size are strong and independent negative prognostic factors for early breast cancer [8]. Tumor diameter and lymph node status were found to act as additive but independent prognostic factors. In the Surveillance, Epidemiology, and End Results (SEER) program of the National Cancer Institute data, 24,740 breast cancer cases were investigated and 5 -year breast cancer survival was up to $67.2 \%$ in patients with tumor size less than $2 \mathrm{~cm}$ and four or more positive lymph nodes, whereas 5 -year breast cancer survival was only $45.5 \%$ in patients with tumors greater than $5 \mathrm{~cm}$ and four or more positive lymph nodes [9]. Although T-stage and nodal status well distributed in both arms in FACE and MA.27 trials, tumor-node-metastases stage, which can effect DFS, may not be very well distributed in both arms.

Adjuvant chemotherapy was given to $31 \%$ of patients in both group in MA.27 trial, whereas adjuvant chemotherapy was administered to 62.7 and $61.1 \%$ of patients in letrozole and anastrozole arms, respectively, in FACE trial $[7,8]$. In a noninferiority Cancer and Leukemia Group B
40101 (NCT00041119) trial, Shulman et al. investigated whether single-agent paclitaxel was inferior to antracycline combination in early breast cancer patients [10]. Multivariable proportional hazards showed that paclitaxel was inferior to antracycline combination on relapse-free survival (RFS) and overall survival (OS), after adjusting for the effects of tumor size, number of positive nodes with an estimated absolute advantage of antracycline combination at 5 years is $3 \%$ for RFS and $1 \%$ for OS [10]. In the 10 -year follow-up of Phase III, randomized BCIRG 001 (NCT00688740) trial, adjuvant anthracycline chemotherapy with taxane combination chemotherapy compared with standard adjuvant antracycline regimen in women with operable nodepositive breast cancer significantly improved both DFS and OS [11]. In another Phase III trial (NCT00004125), the efficacy of two different taxanes, docetaxel and paclitaxel, given either weekly or every 3 weeks, were compared in the adjuvant treatment node positive or high-risk node-negative breast cancer, weekly paclitaxel after standard adjuvant chemotherapy with doxorubicin and cyclophosphamide improved DFS and OS significantly compared with other treatment arms [12]. In a meta-analyses of 13 randomized trials and 22,903 patients with breast cancer, the addition of taxanes to an anthracycline-based chemotherapy significantly improved DFS and OS in high-risk early breast cancer patients [13]. The DFS benefit was also independent in the degree of nodal involvement, type of taxane and administration schedule. Although the rates of adjuvant chemotherapy administrations were similar in both FACE and MA.27 trials, the type of chemotherapy regimens and the type of schedule also can effect the primary outcome DFS.

In FACE trial, about $11 \%$ of patients were also HER2-positive in both arms, adjuvant treatment with trastuzumab was allowed. All four adjuvant randomized trials showed significant benefit of 52 weeks of trastuzumab with taxane plus antracycline combinations in node-positive or high-risk node-negative HER2-positive breast cancer patients [14-16]. In a randomized Phase III North Central Cancer Treatment Group N9831 (NCT00005970) trial, 5-year DFS rate significantly improved from 80.1 to $84.4 \%$ when trastuzumab used concurrently with chemotherapy compared with added sequentially in early-stage HER2-positive breast cancer (HR: 0.77; $p=0.02)[17]$. Thus, the similar distribution 
of HER2 positivity in both treatment arms of FACE trial was not enough to comment that both treatment arms have similar treatment regimens and schedules.

In FACE trial, although no significant DFS difference was reported according to the BMI, the distribution of patients according to the BMI was not reported. In ALIQUOT (Anastrozole vs Letrozole, an Investigation of Quality Of Life and Tolerability) study, baseline plasma estradiol and estrone sulfate levels were significantly correlated with BMI and baseline estradiol values were nearly three-times higher in women with BMI greater than $35 \mathrm{~kg} / \mathrm{m}^{2}$ compared with BMI less than $25 \mathrm{~kg} / \mathrm{m}^{2}$ [18]. This study also revealed that letrozole leads to more complete inhibition of whole-body aromatase compared with anastrozole, and that letrozole induced significantly greater suppression of both estradiol and estrone compared with anastrozole $[5,18]$. In obese patients, the HR for DFS was 0.86, which favors letrozole in patients with $\mathrm{BMI} \geq 35 \mathrm{~kg} / \mathrm{m}^{2}(0.54-1.37)$ compared with anastrozole. The authors present their data based on baseline BMI, but this does not reflect the possibility that some previously 'normal' BMI women became overweight or obese during the 5 years of follow-up. In short, it would be interesting to the reader and pertinent to the analysis to examine whether BMI remained stable during follow-up.

In that respect, it is not easy to comment that all aromatase inhibitors have similar efficacy and safety results before the distribution of unknown confounding factors in both trials.

\section{Financial \& competing interests disclosure}

The authors have no relevant affliations or financial involvement with any organization or entity with a financial interest in or financial conflict with the subject matter or materials discussed in the manuscript. This includes employment, consultancies, honoraria, stock ownership or options, expert testimony, grants or patents received or pending, or royalties.

No writing assistance was utilized in the production of this manuscript

\section{References}

1 Early Breast Cancer Trialists' Collaborative Group. Tamoxifen for early breast cancer: an overview of the randomised trials. Lancet 351(9114), 1451-1467 (1998).

2 Dowsett M, Cuzick J, Ingle J et al. Meta-analysis of breast cancer outcomes in adjuvant trials of aromatase inhibitors versus tamoxifen. J. Clin. Oncol. 28(3), 509-518 (2010).

3 Early Breast Cancer Trialists' Collaborative G, Dowsett M, Forbes JF et al. Aromatase inhibitors versus tamoxifen in early breast cancer: patient-level meta-analysis of the randomised trials. Lancet 386(10001), 1341-1352 (2015).

4 Burstein HJ, Prestrud AA, Seidenfeld J et al. American Society of Clinical Oncology clinical practice guideline: update on adjuvant endocrine therapy for women with hormone receptor-positive breast cancer. J. Clin. Oncol. 28(23), 3784-3796 (2010).

5 Geisler J, Haynes B, Anker G, Dowsett M, Lonning PE. Influence of letrozole and anastrozole on total body aromatization and plasma estrogen levels in postmenopausal breast cancer patients evaluated in a randomized, cross-over study. J. Clin. Oncol. 20(3), 751-757 (2002).

6 Ellis MJ, Suman VJ, Hoog J et al. Randomized Phase II neoadjuvant comparison between letrozole, anastrozole, and exemestane for postmenopausal women with estrogen receptor-rich stage 2 to 3 breast cancer: clinical and biomarker outcomes and predictive value of the baseline PAM50-based intrinsic subtype-ACOSOG Z1031. J. Clin. Oncol. 29(17), 2342-2349 (2011).

7 Goss PE, Ingle JN, Pritchard KI et al. Exemestane versus anastrozole in postmenopausal women with early breast cancer: NCIC CTG MA.27 - a randomized controlled Phase III trial. J. Clin. Oncol. 31(11), 1398-1404 (2013).

8 Smith I, Yardley D, Burris $\mathrm{H}$ et al. Comparative efficacy and safety of adjuvant letrozole versus anastrozole in postmenopausal patients with hormone receptor-positive, node-positive early breast cancer: final results of the randomized Phase III femara versus anastrozole clinical evaluation (FACE) trial. J. Clin. Oncol. 35(10), 1041-1048 (2017).

9 Carter CL, Allen C, Henson DE. Relation of tumor size, lymph node status, and survival in 24,740 breast cancer cases. Cancer 63(1), 181-187 (1989).

10 Shulman LN, Berry DA, Cirrincione CT et al. Comparison of doxorubicin and cyclophosphamide versus single-agent paclitaxel as adjuvant therapy for breast cancer in women with 0 to 3 positive axillary nodes:
CALGB 40101 (Alliance). J. Clin. Oncol. 32(22), 2311-2317 (2014).

11 Mackey JR, Martin M, Pienkowski T et al. Adjuvant docetaxel, doxorubicin, and cyclophosphamide in node-positive breast cancer: 10 year follow-up of the Phase III randomised BCIRG 001 trial. Lancet Oncol. 14(1), 72-80 (2013).

12 Sparano JA, Wang M, Martino S et al. Weekly paclitaxel in the adjuvant treatment of breast cancer. N. Engl. J. Med. 358(16), 1663-1671 (2008).

13 De Laurentiis M, Cancello G, D'agostino D et al. Taxane-based combinations as adjuvant chemotherapy of early breast cancer: a meta-analysis of randomized trials. J. Clin. Oncol. 26(1), 44-53 (2008).

14 Perez EA, Romond EH, Suman VJ et al. Four-year follow-up of trastuzumab plus adjuvant chemotherapy for operable human epidermal growth factor receptor 2-positive breast cancer: joint analysis of data from NCCTG N9831 and NSABP B-31. J. Clin. Oncol. 29(25), 3366-3373 (2011).

15 Slamon D, Eiermann W, Robert N et al. Adjuvant trastuzumab in HER2-positive breast cancer. N. Engl. J. Med. 365(14), 1273-1283 (2011).

16 Piccart-Gebhart MJ, Procter M, LeylandJones B et al. Trastuzumab after adjuvant chemotherapy in HER2-positive breast 
COMMENTARY Şendur, Bilgin, Hızal et al.

cancer. N. Engl. J. Med. 353(16), 1659-1672 (2005).

17 Perez EA, Suman VJ, Davidson NE et al. Sequential versus concurrent trastuzumab in adjuvant chemotherapy for breast cancer. J. Clin. Oncol. 29(34), 4491-4497 (2011).

18 Folkerd EJ, Dixon JM, Renshaw L, A'hern RP, Dowsett M. Suppression of plasma estrogen levels by letrozole and anastrozole is related to body mass index in patients with breast cancer. J. Clin. Oncol. 30(24), 2977-2980 (2012). 